\title{
Serum Proteomic Analysis in Esophagectomy Patients with Postoperative Delirium: A Case-Control Study
}

Walter Fitschen ${ }^{2}$, Sikandar H Khan ${ }^{1,2^{*}}$, Heidi Lindroth ${ }^{1,2,3,4}$, Sophia Wang ${ }^{5}$, Sundus Imran², $^{2}$ Jason D. True ${ }^{6,7}$, Uma Aryal ${ }^{8}$, Anthony J. Perkins ${ }^{9}$, Sujuan Gao ${ }^{9}$, Gideon A. Caplan ${ }^{10,11}$, Homer L. Twigg III ${ }^{1}$, Kenneth Kesler ${ }^{12}$, Babar A. Khan ${ }^{1,2,3}$

${ }^{1}$ Division of Pulmonary, Critical Care, Sleep and Occupational Medicine, Department of Medicine, Indiana University School of Medicine, Indianapolis, Indiana, USA; ${ }^{2}$ Indiana University Center for Aging Research, Regenstrief Institute, Indianapolis, Indiana, USA; ${ }^{3}$ Indiana University Center of Health Innovation and Implementation Science, Indianapolis, Indiana, USA; ${ }^{4}$ Indiana University School of Nursing, Indianapolis, Indiana, USA; ${ }^{5}$ Department of Psychiatry, Indiana University School of Medicine, Indianapolis, Indiana, USA; ${ }^{6}$ Department of Biochemistry and Molecular Biology, Indiana University School of Medicine, Indianapolis, Indiana, USA;

${ }^{7}$ Department of Biology, Ball State University, Muncie, Indiana, USA; ${ }^{8}$ Purdue Proteomics Facility, Bindley Bioscience Center, Purdue University, West Lafayette, Indiana, USA;

${ }^{9}$ Department of Biostatistics, Indiana University School of Medicine, Indianapolis, Indiana, USA; ${ }^{10}$ Prince of Wales Clinical School, University of New South Wales, Sydney, New South Wales, Australia; ${ }^{11}$ Department of Geriatric Medicine, Prince of Wales Hospital, Sydney, New South Wales, Australia; ${ }^{12}$ Department of Cardiothoracic Surgery, Indiana University School of Medicine, Indianapolis, Indiana, USA

Background: Delirium is a common post-operative complication for patients who undergo esophagectomy. The pathophysiology of delirium is poorly understood, limiting therapeutic options. To advance our pathophysiologic understanding, we conducted this study to evaluate the association of protein expression with postoperative delirium.

Methods: A case-control study was performed using blood samples from the PEPOD randomized clinical control trial (NCT02213900). 52 patients (26 cases with delirium, 26 control) who were $>18$ years old, spoke English, and underwent esophagectomy were included, while those with schizophrenia, Parkinson's, alcohol abuse, neuroleptic malignant syndrome, 
haloperidol allergy, and severe dementia were excluded. Samples were from pre-op and postop day 1. Delirium was assessed for using the Confusion Assessment Method for the Intensive Care Unit and Richmond Agitation Sedation Scale, while the severity was assessed using DRS-R68. Proteomic analysis was performed using TMT 10ples isobaric labels. Proteome Discoverer and STRING web-based tools were used for bioinformatic analyses, and the Wilcoxon-MannWhitney Odds (WMWodds) test was used to report between-group differences in protein abundance ratios.

Results: 52 samples (26 cases, 26 controls) were analyzed for this study. Elevated protein abundance ratios of Coagulation factor IX (Median (IQR): $1.18(1.08-1.28)$ p=0.047) and Mannosyl-oligosaccharide 1,2-alpha-mannosidase (Median (IQR): 1.50 (1.35-1.60) p=0.049) were noted in patients that developed delirium. Elevated protein abundance ratios of angiotensinogen (Median (IQR): 1.19 (1.14-1.23) p=0.038), ceruloplasmin (Median (IQR): 1.02 (0.98-1.08) p=0.038), and Complement C2 (Median (IQR): 1.01 (0.96-1.08) p=0.016) were noted in control patients. Protein abundance ratios for Complement C2 (Spearman $r_{s}=0.31,95 \% \mathrm{Cl}$ [$0.55,-0.02])$ and Mannosyl-oligosaccharide 1,2-alpha-mannosidase $\left(r_{s}=0.61,95 \% \mathrm{Cl}[0.29,0.81]\right)$ were associated with the severity of delirium in case patients.

Conclusions: Changes in proteins associated with coagulation, inflammation, and protein handling were identified. These could be evaluated in future studies to develop tools for predicting onset and severity of post-operative delirium. 